\title{
An Indoor AC Magnetic Positioning System
}

\author{
Guido De Angelis ${ }^{1}$, Valter Pasku ${ }^{1}$, Alessio De Angelis ${ }^{2}$, Marco Dionigi ${ }^{1}$, Mauro Mongiardo ${ }^{1}$, \\ Antonio Moschitta ${ }^{1}$, Paolo Carbone ${ }^{1}$ \\ ${ }^{1}$ Department of Engineering, University of Perugia, \\ Via G. Duranti 93, 06125 Perugia, Italy \\ ${ }^{2}$ Department of Engineering, Polo Scientifico e Didattico di Terni, University of Perugia, \\ Strada di Pentima 4, 05100 Terni, Italy \\ guidodeangelis@ieee.org, valter.pasku@studenti.unipg.it \{alessio.deangelis, marco.dionigi, mauro.mongiardo, \\ antonio.moschitta, paolo.carbone\}@unipg.it
}

\begin{abstract}
This paper describes the design and realization of a Magnetic Indoor Positioning System. The system is entirely realized using off-the-shelf components and is based on inductive coupling between resonating coils. Both system-level architecture and realization details are described along with experimental results. The realized system exhibits a maximum positioning error of less than $10 \mathrm{~cm}$ in an indoor environment over a $3 \times 3 \mathrm{~m}^{2}$ area. Extensive experiments in larger areas, in non-line-of-sight conditions, and in unfavorable geometric configurations, show sub-meter accuracy, thus validating the robustness of the system with respect to other existing solutions.
\end{abstract}

Keywords - Indoor positioning, electromagnetic analysis, inductive coupling, mutual coupling, Magnetic Indoor Positioning System.

\section{INTRODUCTION}

Estimating the position of a user where it is not possible to use the GPS system, such as in indoor environments, is a common issue in many practical applications [1][2]. In recent years, therefore, the interest for developing indoor positioning systems has been increasing. Several kinds of indoor localization technologies were applied for estimating user position, including Ultra Wide Band (UWB) [3], ZigBee [4], RFID, Bluetooth, ultrasound, laser; for an extensive survey, see $[5]$.

The lack of accuracy caused by multipath propagation or constructive complexity has led to new solutions such as Magnetic Indoor Positioning Systems (MIPS) [6]-[8]. In this context, in fact, systems based on magnetic fields have the desirable property of being robust to multipath, of being easy to produce and of having the ability to penetrate many materials. In [7] and [8], the use of direct-current (DC) artificiallygenerated magnetic fields was investigated. This approach allows user self-localization by using exclusively a tri-axis magnetometer. However, this approach has the disadvantage of requiring a considerable current consumption, $12 \mathrm{~A}$, for the generation of the static magnetic field. Furthermore, for achieving a usable range of about $18 \mathrm{~m}$, it requires costly highsensitivity sensors.

In [9], an alternative approach was proposed for distancemeasurement based on magnetic fields. In particular, two resonant mutually coupled circuits with a low frequency alternating current (AC) signal were considered. Due to inductive coupling and high resonator quality factors, this system enables a more efficient operation by decreasing the required current for a given operating range, with respect to the DC approach. The main advantage of the magnetic based technique is the improved robustness with respect to multipath phenomena in comparison to radio-frequency (RF) based solutions. Magnetic fields, in fact, are relatively less sensitive to the propagation-dependent disturbances that affect RF systems. Furthermore, the excellent material penetration properties make this technology a suitable candidate for use in harsh non-line-of-sight (NLOS) conditions. In such conditions, in fact, some competing technologies are not applicable, e.g. laser and ultrasound, while others are subject to performance degradation, see e.g. the UWB system in [10]. Moreover, thanks to the resonance property, the proposed system allows for higher operational range and lower current consumption with respect to DC magnetic field solutions, thus potentially enabling novel applications to longer-range and building-wide positioning scenarios. Finally, the proposed approach has the potential of low-complexity and low-cost implementation, as proven by the realized prototype, which is built using off-theshelf discrete components.

In this paper, the basic principle of AC magnetic onedimensional ranging described in [9] is extended to realize a planar indoor positioning system. To the authors' knowledge, this approach has not been deeply investigated in the scientific literature. In fact the basic principle is exploited in existing commercial systems such as that in [6], where however resonance does not appear to be employed. The usage of coplanar resonant coils for positioning purposes has been considered in [11], but the considered range is limited to 30 $\mathrm{cm}$, it relies on the impedance analysis of a relay antenna, interacting with the power transfer between two coils, and it requires a network analyzer. The system proposed in this paper features a much higher range, tested up to approximately $8 \mathrm{~m}$ in an NLOS indoor environment, and features a simpler and less expensive architecture, since it does not use a relay antenna. Moreover, it is based on received power measurements, rather than on impedance measurements. 


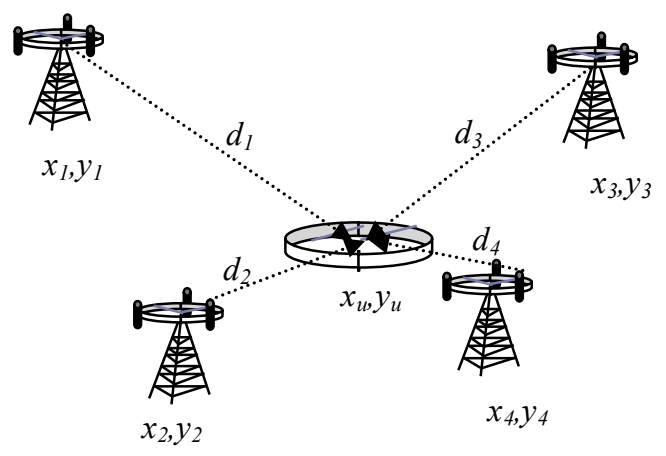

Fig. 1 - Architecture of the considered magnetic positioning system.

In [12], the basic hardware configuration of the positioning prototype system was presented. In this paper, we describe a complete developed system, addressing several implementation issues such as bandwidth and frequency separation operating mode. Furthermore, we provide an extensive characterization in a variety of environments, showing the robustness of the system under typical and harsh indoor conditions.

\section{SYSTEM DESIGN}

The architecture of the realized system is shown in Fig. 1. It is based on a network of transmitter nodes, which are placed at known positions. Each transmitter node consists of an oscillator circuit and of a tuned resonating coil. Additionally, the network contains a receiver node, the position of which is to be estimated, tuned to the same nominal frequency. Each transmitter coil generates an electromagnetic field that interacts with the receiver coil, by inducing a current.

The received root-mean-square voltage, $V_{R x}$, at the output of the receiver resonating circuit, may be obtained as [9]:

$$
V_{R x}=V_{T x} \cdot k \sqrt{Q_{1} Q_{2}}
$$

where $V_{T x}$ is the transmitted root-mean-square voltage, $k$ is the coupling factor, $Q_{1}$ and $Q_{2}$ denote the resonator quality factors of the transmitter and receiver respectively, with $Q=\omega L / R$. The coupling factor can be expressed as $k=M / \sqrt{L_{1} L_{2}}$, where $M$ is the mutual inductance, $L_{1}$ is the transmitter coil inductance and $L_{2}$ is the receiver coil inductance.

The mutual inductance $M$ is, in general, considerably difficult to compute. However, as we show in Section III, under some specific configurations, $M$ depends only on the distance between the coils. Therefore, by measuring $V_{R x}$ at the receiver, it is possible to relate the voltage to the distance, as shown experimentally in Section IV. Finally, in the presence of at least three transmitter nodes, the receiver position in two dimensions can be estimated by using trilateration algorithms.

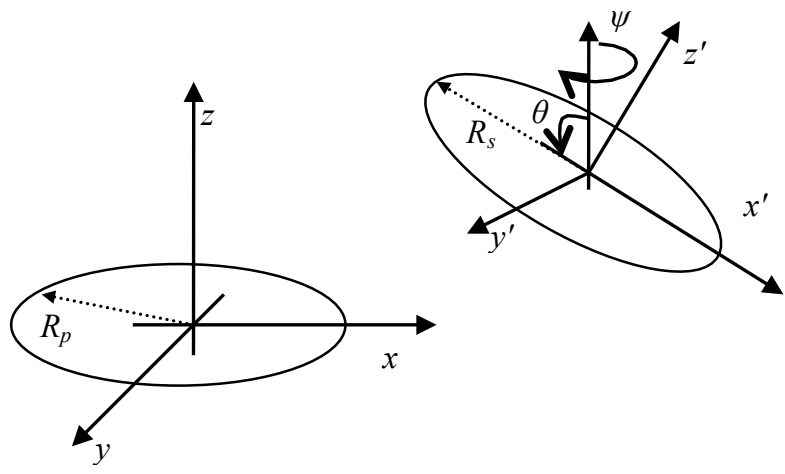

Fig. 2 - Filamentary circular coils with arbitrary lateral and angular misalignment [13].

\section{ESTIMATION OF THE MUTUAL INDUCTANCE}

Provided that the dependence of $k$ and $M$ on the distance $d$ is known, $d$ can be estimated by measuring $V_{R x}$ at the receiver, using (1). The mutual inductance $M$ is related to the coupling factor $k$, that depends both on the coils' characteristics and on the reciprocal position and orientation of the coils. Even if such parameters and the model relating them to the mutual inductance are known, the evaluation of $M$ in an arbitrary coil configuration is quite complex, as well as univocally relating $M$ to the distance $d$ between the coils. However, as shown in the following, in the coplanar coil configuration $M$ only depends on $d$. Therefore, a planar position could be estimated by performing $V_{R x}$ measurements between at least three beacons and the mobile node, using (1) to estimate a set of distances and by subsequently performing trilateration.

We start by analyzing the general case. Consider two circular filaments of radii $R_{P}$ and $R_{S}$ as shown in Fig. 2. The center of the primary filament is located at the origin of the coordinate system. The secondary filament of radius $R_{S}$ is located in an inclined plane $\lambda$ whose general equation is given by

$$
\lambda \equiv a x+b y+c z+d=0
$$

The parameters in (2) can be expressed in spherical coordinates using the following transformation laws,

$$
\begin{aligned}
& a=\sin \psi \sin \theta \\
& b=-\cos \psi \sin \theta \\
& c=\cos \theta
\end{aligned}
$$

Given the above equivalence, the mutual inductance can be obtained for various $\psi$ angles by using the following expression [13]:

$$
M=\frac{\mu_{0} R_{S}}{\pi} \int_{0}^{2 \pi} \frac{\left[p_{1} \cos \varphi+p_{2} \sin \varphi+p_{3}\right] \Psi(k)}{k \sqrt{V_{0}^{3}}} d \varphi
$$




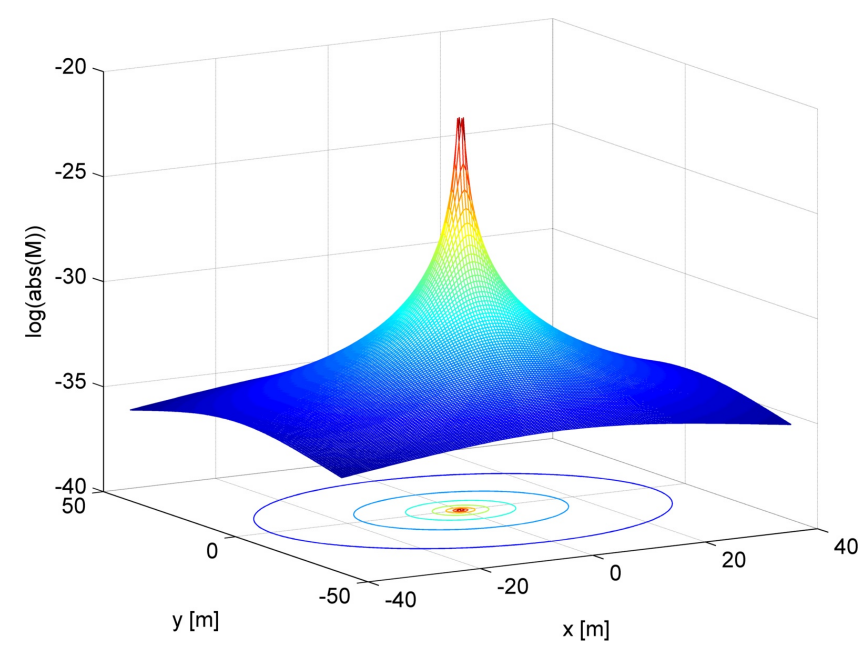

Fig. 3 - Numerical simulation results for the mutual inductance $M$ between two coplanar filaments, calculated according to Eq. (4), as a function of the secondary filament position $(\mathrm{x}, \mathrm{y})$. Here the primary filament is assumed to be located at $(0,0)$.

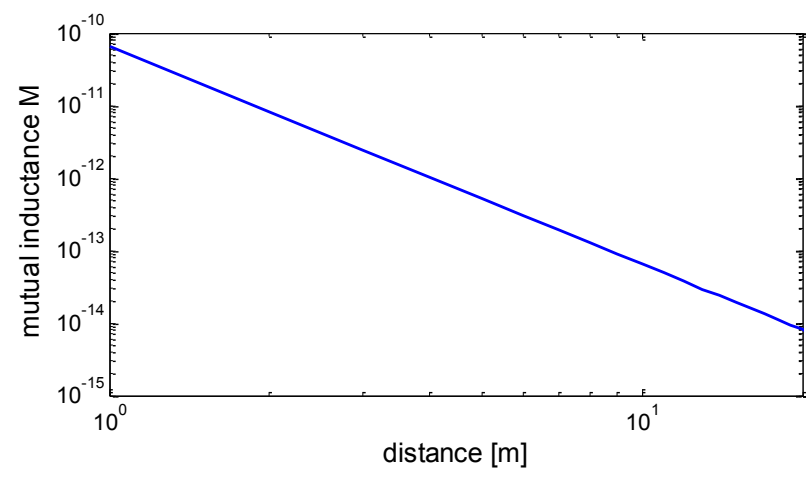

Fig. 4 - Numerical simulation results for the mutual inductance $M$ between two coplanar filaments, calculated according to Eq. (4), as a function of the distance between the centers of the two filaments.

In the case of two coplanar filaments, i.e. when the transmitter and receiver filaments are located in the same plane, we have that $M$ has a circular symmetry on the $x y$ plane $(c z+d=0)$, as shown in Fig. 3. The plot in this figure was obtained by numerical integration of (4) on a grid of coordinates in the $x y$ plane, using the Matlab numerical computation environment. The coil radii and the other physical parameters in the simulation have been set to the same numerical values as those of the realized system prototype and are provided in Section $\mathrm{V}$.

Furthermore, in Fig. 4, the behavior of $M$ as a function of $d$ is shown, as computed numerically according to (4) in the coplanar coil configuration. On a bilogarithmic scale, this behavior is linear, implying that the system calibration can be performed by linear fitting. In the following section, we provide experimental results supporting such claim.

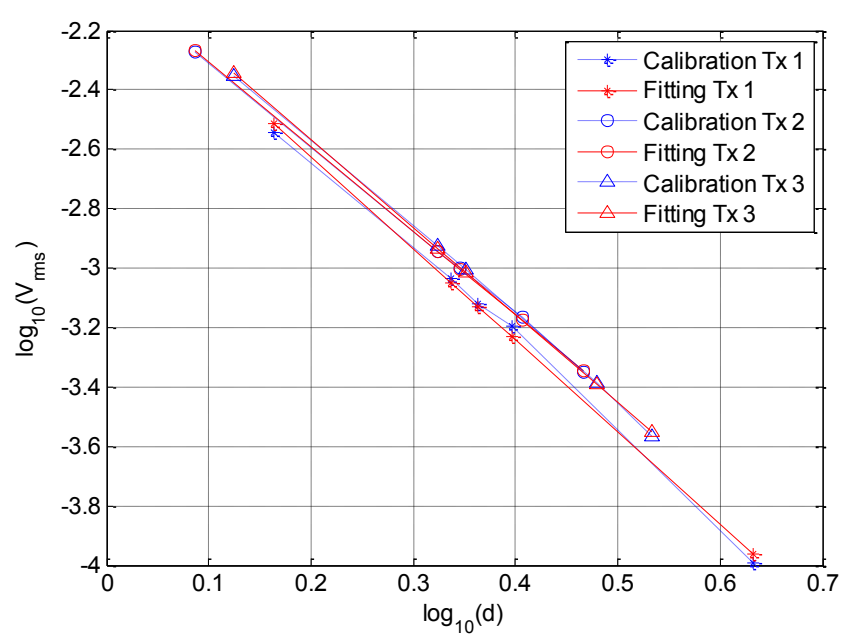

Fig. 5 - Experimental calibration results, obtained for 3 transmitter nodes (Tx1, Tx2, and Tx3). The received root-mean-square voltage, expressed in V, is plotted as a function of distance, expressed in $\mathrm{m}$.

Moreover, we note that the coplanar configuration is an advantageous feature. In [9], in fact, the one-dimensional ranging was performed by using a coaxial configuration of the coils. Such a configuration is not feasible for $2 \mathrm{D}$ positioning applications, given the different orientations of multiple transmitter coils relative to a receiver coil. Furthermore, any configuration other than the coplanar one would cause the behavior of $M$ to be anisotropic and thus would require a complex procedure for estimation of the relative orientation in order to measure the distance.

TABLE I: Experimental calibration parameters, obtained by linear fitting of the data in Fig. 5.

\begin{tabular}{|c|c|c|}
\hline & Intercept & Slope \\
\hline Transmitter 1 (Tx 1) & -2.0046 & -3.0991 \\
\hline Transmitter 2 (Tx 2) & -2.0262 & -2.8248 \\
\hline Transmitter 3 (Tx 3) & -1.9765 & -2.9474 \\
\hline
\end{tabular}

\section{POSITION MEASUREMENT}

The proposed position measurement procedure can be divided into two distinct phases, namely calibration and trilateration. The first phase is necessary to characterize the behavior of each individual node. In fact, every node has special characteristics related to its realization, e.g. component tolerances and amplifier gain differences, and to environmental configurations, e.g. the presence of conductive objects. The characterization of each node is required to convert the measured voltage to distance and is aimed at reducing position estimation inaccuracies. The second phase consists of the estimation of the user position. 


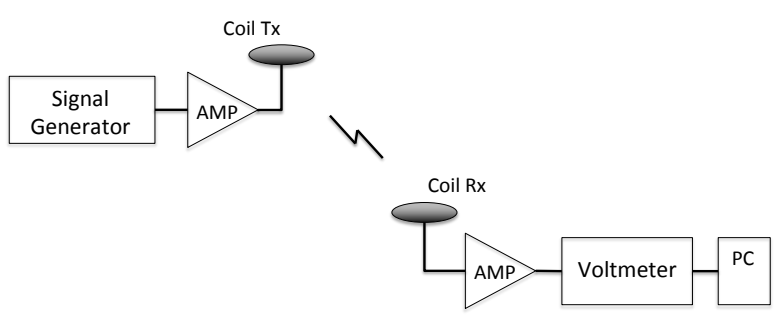

Fig. 6 - Block diagram of the realized prototype.

The calibration phase needs to be executed only once during the initial deployment of the system in a given environment. During the calibration phase, the receiver node is placed at a set of reference distances from each transmitter node. For every reference distance, denoted by $d$, a measurement of $V_{r m s}$ is performed. Experimental results from the calibration phase using the realized prototype system are shown in Fig. 5. To generate this plot, reference distances between $1.22 \mathrm{~m}$ and $4.29 \mathrm{~m}$ were used. The transmitter and receiver nodes were placed in line-of-sight conditions in a typical indoor office environment. This plot shows an approximately linear behavior on a bilogarithmic scale, that suggests the use of the linear fitting method to obtain the calibration parameters, reported in Table I. Such linear behavior is consistent with the expected theoretical behavior described in the previous section.

During the trilateration phase, by processing measurements of the received voltages from $n$ transmitter nodes, with $n \geq 3$, it is possible to estimate the receiver's position. Specifically, we consider a 2-D localization problem, and we denote as $\xi=\left[\begin{array}{ll}x & y\end{array}\right]^{T}$ the column vector of the unknown coordinates of the receiver. The known coordinates of the $i$-th transmitter are denoted by $\left(x_{i}, y_{i}\right)$. The distance $\delta_{i}$ between the transmitter and the $i$-th receiver is given by

$$
\delta_{i}=\sqrt{\left(x-x_{i}\right)^{2}+\left(y-y_{i}\right)^{2}} \quad i=1,2, \ldots n
$$

Defining the vector of measured transmitter-receiver distances as $D=\left[d_{1} d_{2} \ldots d_{n}\right]^{T}$, the nonlinear least squares (NLS) method can be adopted to find the value $\hat{\xi}$ of $\xi$ that minimizes the sum of squared errors

$$
J=[D-\Delta(\xi)]^{T}[D-\Delta(\xi)]
$$

where $\Delta(\xi)=\left[\delta_{1} \delta_{2} \ldots \delta_{n}\right]^{T}$.

The NLS algorithm cycles over a fixed number of iterations. Repeated empirical tests suggested that 6 cycles are enough for the algorithm to converge with $n=3$.

\section{EXPERIMENTAL RESULTS}

The realized system prototype consists of a set of nominally identical resonators, implemented by coils having two turns each and a radius of $0.09 \mathrm{~m}$. The coils feature an inductance $L \cong 1.54 \mu \mathrm{H}$, measured with a RLC meter, while the coils' resonator $Q$ factors equal 857 . A block diagram of a two coil prototype is shown in Fig. 6. One of the coils is configured as a transmitter, and is connected to a benchtop signal generator programmed to provide a sinusoidal signal with a peak-to-peak amplitude of $10 \mathrm{~V}$ and a frequency of

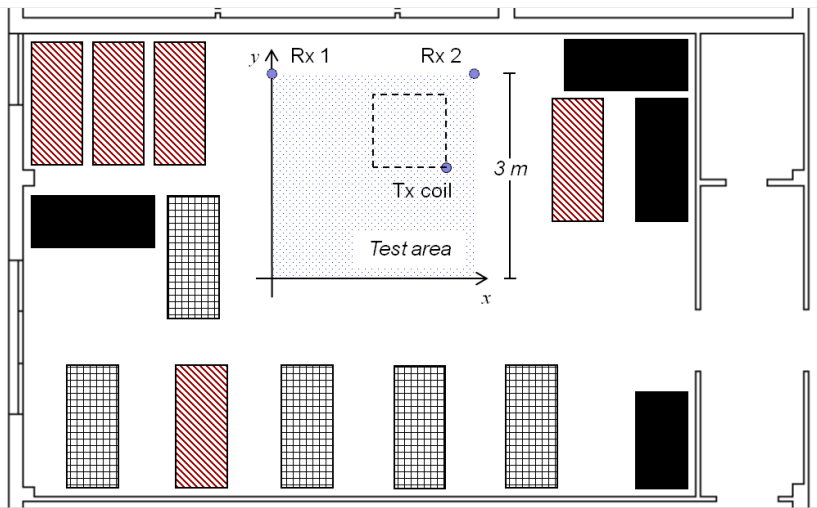

Fig. 7 - Laboratory setup for the preliminary experimental test. Solid metal cabinets are denoted by solid black rectangles, wooden benches by diagonal dashed patterns, and metallic-frame benches by gridded patterns.

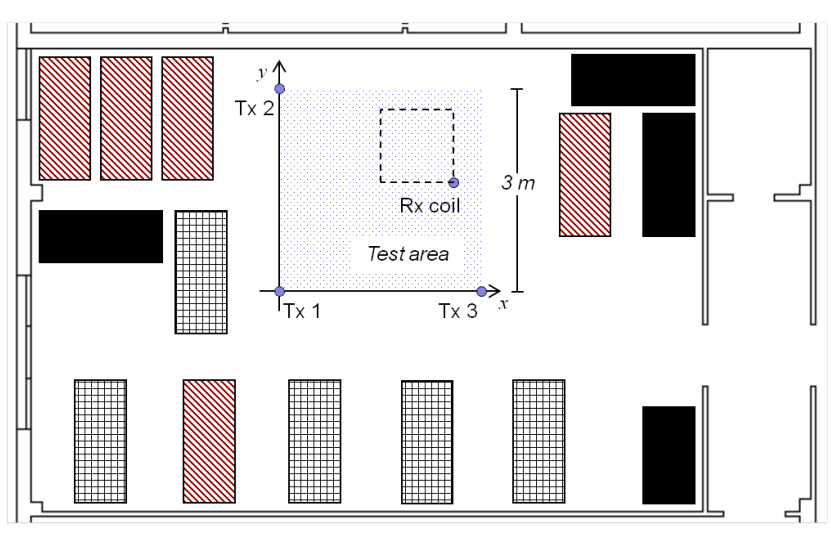

Fig. 8 - Laboratory setup for the second experiment. Three transmitting coils have been deployed as beacons, and the mobile node acts as a receiver.

$189.5 \mathrm{kHz}$. Therefore, we have that $V_{T x}=3.53 \mathrm{~V}$. Further, an OPA549 amplifier is connected to the output of the signal generator, to drive the transmitting coil.

The other coil is configured as a receiver, and is connected to the input of an instrumentation amplifier (INA) integrated circuit, the AD8421 by Analog Devices, configured to provide a gain $G=100$. The root-mean-square voltage at the output of the instrumentation amplifier is measured with a true-rms digital multimeter.

During the experimental tests, the transmitter and receiver coils were placed in a coplanar configuration at known positions in a laboratory environment. A floorplan of the laboratory used for the experiments is shown in Figs. 7 and 8, illustrating the presence of walls and conductive objects such as metal cabinets and wooden benches with metallic frames.

In order to get the reference positions in the test configurations, the following procedure was employed: 1. Reference points were marked on the floor, their positions were measured with a tape measure and exploiting the alignment of the floor tiles. 2. The coils were placed on inhouse built stands ensuring that the center of each coil was aligned with the base of the related stand. This base was then placed directly on the marked point. 3. Acquisitions were made at the marked points. 


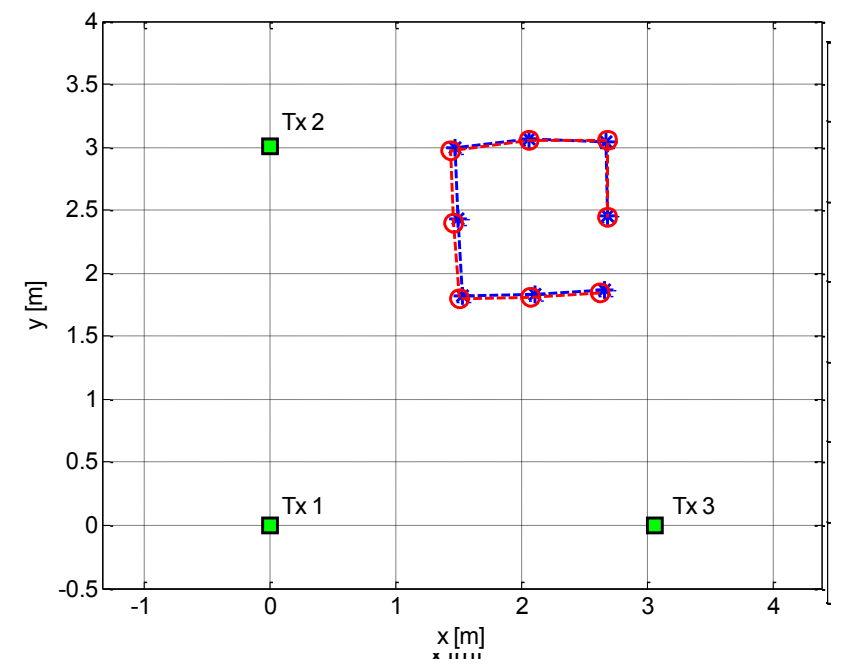

Fig. 9 - Indoor positioning experimental results. The known transmitter positions are denoted by green squares, the true receiver positions by red circles and the estimated receiver positions by blue asterisks.

\section{A. Preliminary Test}

In a preliminary phase, an experimental test was performed, using the first two available nodes of the prototype shown in Fig. 6. In this configuration, the transmitting coil acted as mobile node, and the receiving coil has been repeatedly placed in various know positions. Such configuration mimics the usage of $n$ receivers, placed at fixed and known positions, while the goal is to locate the transmitter node by processing the measurements of the received voltages. Note that, the theoretical considerations of Section III and IV still apply, and the position can be estimated using the algorithm presented in Section IV.

During the preliminary experimental test, the laboratory configuration shown in Fig. 7 was employed. The receiver coil was placed in two positions sequentially, namely Rx1 and $\mathrm{Rx} 2$. For each receiver position, the transmitter coil was placed in four positions at the corners of a $1 \mathrm{~m} \times 1 \mathrm{~m}$ square, in the middle region of the laboratory. A calibration procedure equivalent to that described in Section IV was carried out. Since using only two beacons in planar trilateration leads to two possible solutions, a priori knowledge of the environment has been used to discriminate the correct solution. The positioning error has been evaluated as the Euclidean distance between each estimated mobile node position and the corresponding ground-truth position. The measurement results exhibit a maximum error of approximately $1.5 \mathrm{~cm}$ and an average error of approximately $0.7 \mathrm{~cm}$. This good accuracy has been ascribed to the fact that that the transmitter is placed in a favorable condition, i.e. the middle of the laboratory, where the influence of environmental effects is not prominent.

\section{B. Performance Characterization}

Following such results, the more realistic setup of Fig. 8 was deployed, featuring three beacons to locate the mobile node. Moreover, the role of the available nodes was swapped, using transmitters as fixed beacons and a receiver as mobile node. Such a solution may be more suitable for safety reasons,

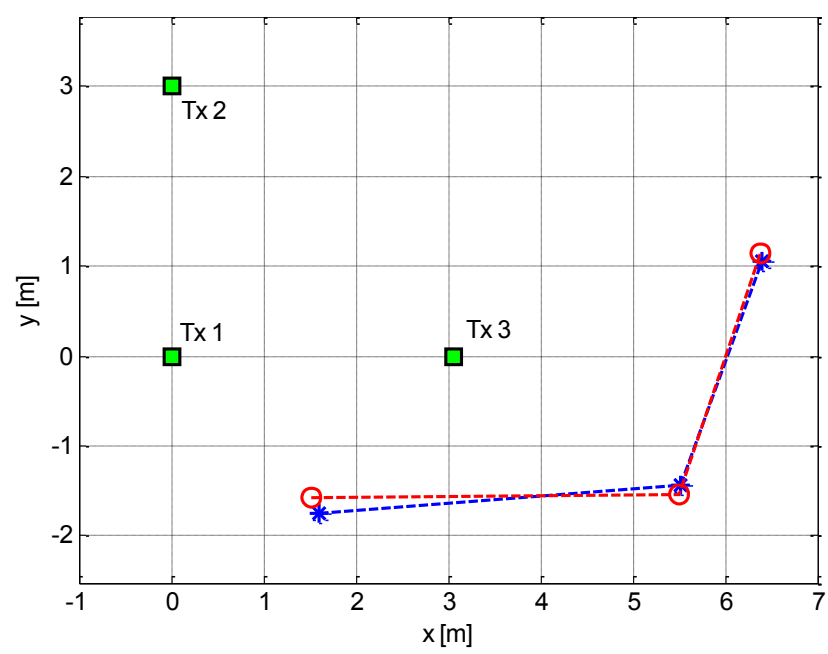

Fig. 10 - Experimental results for larger environment configuration. The known transmitter positions are denoted by green squares, the true receiver positions by red circles and the estimated receiver positions by blue asterisks.

if the mobile node is to be used by a human operator, since it reduces the local magnetic field. Moreover, it may increase the operational life of a battery powered mobile node, shifting the magnetic field generation task to the beacons.

In particular, the three transmitter coils were placed in three of the corners of a $3 \times 3 \mathrm{~m}^{2}$ square, as shown in Fig. 9, while the receiver coil was placed in eight positions in a $1.2 \times 1.2 \mathrm{~m}^{2}$ area. For each position, the ground-truth values were measured and recorded, for comparison with the trilateration estimates. Under such condition, an average error of $3.3 \mathrm{~cm}$ was obtained, with a maximum error of about 5.3 $\mathrm{cm}$. Such results prove the good potential of the proposed method, provided that the fixed beacons suitably surround the area where the mobile node is to move.

However, even when this requirement is not satisfied, the system is still capable of providing reasonably accurate performance. An experiment has been performed in a less favorable configuration to support this claim. In particular, the mobile node was placed at larger distances from the fixed beacons, and in a less favorable situation, shown in Fig. 10, such that the mobile node was out of the square area marked by the fixed beacons, with a maximum transmitter-receiver distance of approximately $7 \mathrm{~m}$. In this case, an increased average error of $12.7 \mathrm{~cm}$ was obtained, probably due to the deterioration of the geometric dilution of precision. Notice that, even if increased, the error magnitude is still acceptable, and adequate to estimate the position of a person moving in an indoor environment.

To evaluate the maximum operating range, additional tests have been performed. Results have shown that the realized system can reach a maximum transmitter-receiver distance of approximately $20 \mathrm{~m}$ in open-field line-of-sight conditions. If a longer range is required by the application, it can be achieved by increasing the magnitude of the generated AC magnetic field, at the expense of a higher infrastructure power consumption. Moreover, the presence of people in the transmitter-receiver path does not influence the performance, as the receiver readings are not noticeably affected. 
As the transmitter - receiver distance increases, two main aspects become significant, namely, terrain conductivity and the presence of metallic objects in the vicinity of the coil. Terrain conductivity can be compensated by the complex image theory method, as discussed for an outdoor magnetic positioning application in [14]. Regarding the presence of metallic objects, it can be noticed that "hard-iron" disturbances, which are caused by magnets, do not influence the system. In fact, they add a constant DC offset to the magnetic field and thus are rejected by the $\mathrm{AC}$ resonant system at the receiver. On the other hand, "soft-iron" disturbances which are caused by ferromagnetic materials can influence the system. Furthermore, eddy currents induced by the time-varying magnetic field on conducting objects in close proximity to the receiver can also modify the magnetic field measured at the receiver. To quantify such effect, a worst-case experiment was conducted by placing a conductive copper plate directly above the receiving coil. The measured power was $10 \mathrm{~dB}$ below the power received in the absence of the plate. Even if this experiment represents a worst-case condition unlikely to happen in practical applications, it motivates future system development work to improve robustness.

To mitigate the effects of soft-iron and eddy currents phenomena, it is desirable to decrease the operating frequency [15]. However, such a decrease implies a reduction of the resonance quality factor thus reducing the maximum operating range. Therefore, a tradeoff is established between sensitivity to environmental configurations and maximum operating range.

\section{NLOS Performance Characterization}

Furthermore, the robustness of the realized system with respect to environmental configurations, specifically non-lineof-sight (NLOS) conditions, has been evaluated. To this end, the three transmitters were placed outside the laboratory used for the previous experimental characterizations. The mobile receiving node was instead placed inside the laboratory in eight positions in a $1.2 \times 1.2 \mathrm{~m}^{2}$ area, as shown in Fig. 11 . The distance between the transmitters themselves is larger than that of Figs. 9 and 10, e.g. Tx 1 and Tx 2 are approximately 6.5 $\mathrm{m}$ apart, while the maximum transmitter-receiver distance is approximately $8 \mathrm{~m}$. In such configuration, the system is still fully functional, and results show an average error of $13.3 \mathrm{~cm}$. Thus the feasibility of the realized system in through-wall NLOS applications is proven.

This experimental configuration is interesting for practical applications. In fact, it emulates the scenario of a user entering a building without pre-installed positioning infrastructure. In such a scenario, the transmitters can be deployed outside of the building in known positions, i.e. using GPS receivers, and a user carrying the mobile node can maintain seamless outdoor-to-indoor positioning coverage. Potentially, such a scenario is of practical importance for emergency operations, where first responders typically cannot rely on a positioning infrastructure [16].

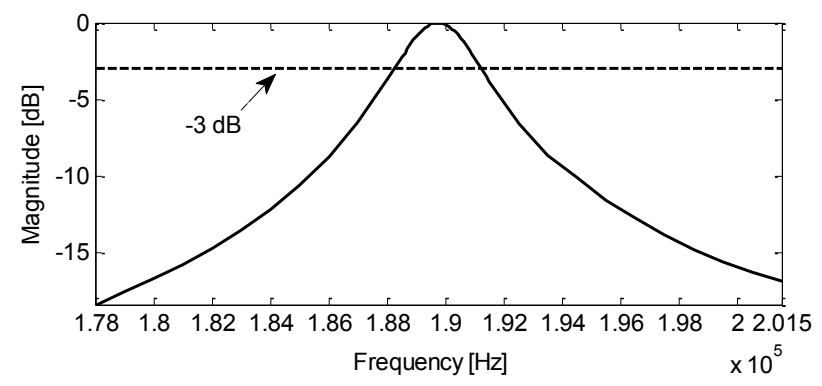

Fig. 12 - Measurement of the system bandwidth. The received power is normalized so that the maximum shown is $0 \mathrm{~dB}$. The $-3 \mathrm{~dB}$ level is denoted by a dashed line.

\section{SYSTEM IMPLEMENTATION ISSUES}

The realized system can operate in two different modes, namely time separation and frequency separation. In time separation mode, the transmitters are enabled in a sequential fashion, such that only one transmitter is operating at any time. Conversely, in frequency separation mode, the transmitters are simultaneously operating in a continuous fashion. In order for the receiver to discriminate between multiple transmitters, a different frequency is assigned to each transmitter. The frequency separation mode allows for a faster measurement update rate, at the expense of an increased peak power consumption.

These two operation modes pose several implementation issues, which will be addressed in the following subsections. As a preliminary step, a characterization of the system bandwidth is provided in the next subsection.

\section{A. Bandwidth Characterization}

The system bandwidth (BW) is a fundamental parameter that needs to be considered for implementation purposes, due to the resonant behavior of the system. An experimental characterization of BW is performed by transmitting at a fixed distance at different frequencies and measuring the received power as shown in Fig. 12.

The received power is measured with an Agilent N9320B $9 \mathrm{kHz}-3 \mathrm{GHz}$ spectrum analyzer using a fixed reference level of $10 \mathrm{dBm}$, span $1 \mathrm{kHz}$, resolution $\mathrm{BW}$ and video $\mathrm{BW}$ of $10 \mathrm{~Hz}$ and number of averages equal to 10 . The center frequency of the instrument is set to the transmitted frequency for any measurement. The fixed distance between transmitting and receiving coil is $3 \mathrm{~m}$ and the transmitted sinusoidal signal has peak-to peak amplitude of $10 \mathrm{~V}$.

The system acts as a band pass filter with center frequency of approximately $189.7 \mathrm{kHz}$ and shows a 3-dB bandwidth of approximately $3 \mathrm{kHz}$. The attenuation of the second and third harmonics of the center frequency, not shown in this figure, is respectively 66 and $81 \mathrm{~dB}$.

The bandwidth characterization provided above is useful to optimize system performance. In time separation mode, in fact, the maximum range can be achieved using the center frequency of the system. In frequency separation mode, the bandwidth provides an upper bound to the interval of frequencies that can be assigned to each transmitter. Moreover, a careful choice of such frequencies should be 


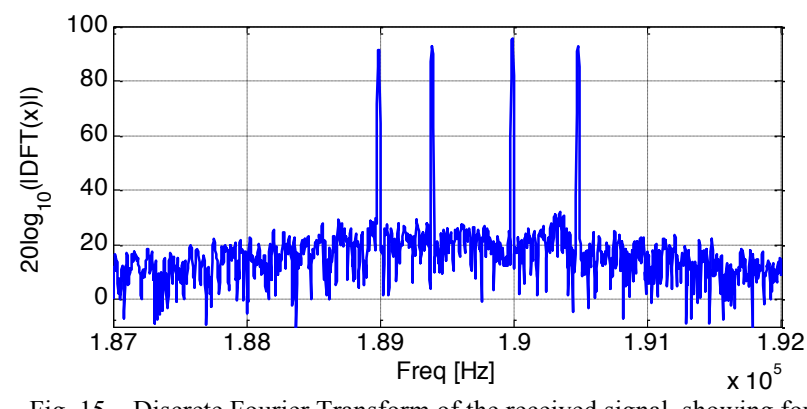

Fig. 15 - Discrete Fourier Transform of the received signal, showing four peaks corresponding to the transmitted sinusoidal signals.

made, in order to avoid interferences. In the next subsection, an analysis of such issue is provided.

\section{B. Frequency Separation Mode Implementation}

The realized system presents a non-linear saturation behavior when the receiver is physically close to a transmitter. When the distance is approximately $1 \mathrm{~m}$ or smaller, in fact, the output voltage swing limit of the INA is reached. This can be partially mitigated by buffering the INA's output or by connecting it to a high-impedance load, but it cannot be completely avoided. Such behavior can influence the measurement results increasing the estimation error. Operating in frequency separation mode, in particular, the receiver input signal is given by the sum of $N$ sinusoidal signals at different frequencies $f_{i}, i=1, \ldots, N$ near the resonance of the system. When this signal is input into a non-linear system, the output signal is corrupted by intermodulation products.

The third-order intermodulation products at frequencies $2 f_{i}-f_{j}$ and $f_{i}+f_{j}-f_{k}$ where $i, j, k=1, \ldots N$ and $i \neq j, i \neq k, j \neq k$, are of particular significance, because such frequencies can overlap the frequencies of interest $f_{i}$ [17]. In this case the signal from a distant beacon can be hidden by one or more intermodulation products and estimations might become biased. A mitigation of this effect is obtained by a proper choice of the frequencies assigned to each transmitter. In particular, denote as $x$ the minimum acceptable frequency difference between a third-order intermodulation product and $f_{i}$. Then, to avoid interference, the following conditions must be satisfied:

$$
\begin{aligned}
& \Delta_{\text {min }} \geq 2 x \\
& \delta_{\text {min }} \geq x
\end{aligned}
$$

where $\Delta_{\min }$ is the minimum separation between frequencies of interest, and $\delta_{\min }$ is the minimum difference between any two separation values. Specifically, we have experimentally observed that using $N=4$ with frequencies chosen in the [189.0 $189.4190 .0190 .5] \quad \mathrm{kHz}$ set, the separation between frequencies of interest and intermodulation products has a minimum value of $100 \mathrm{~Hz}$. This provides a good trade-off between frequency separation and interference level.

Limited available bandwidth is indeed an issue for the scalability of the system. However, exploiting the properties of the DFT, the frequency separation between different transmitters can be reduced at the expense of an increased acquisition length. Apart from this tradeoff, possible solutions

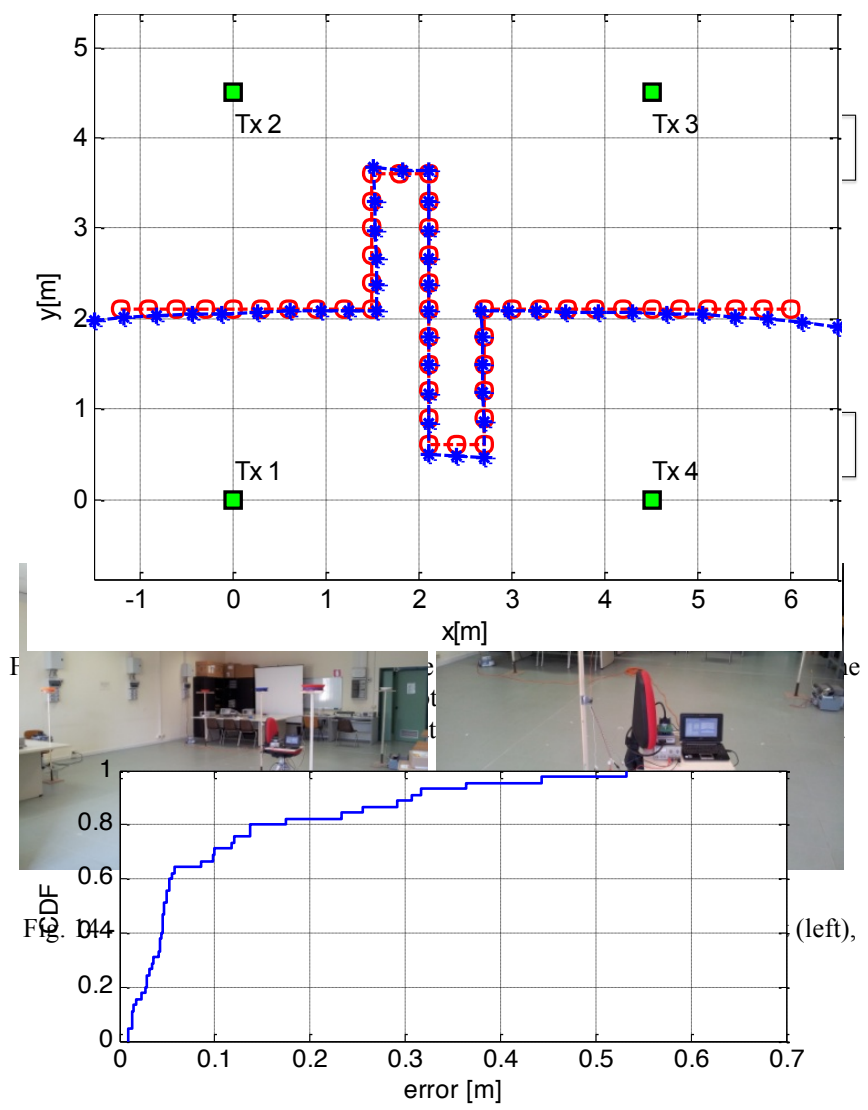

Fig. 17 - Empirical CDF of the positioning error for the trajectory in Fig. 16.

include the implementation of more sophisticated multi-user access techniques, such as frequency hopping or code-division multiple access. Alternatively, the receiver could be equipped with multiple resonators, tuned to different bands assigned to different sets of transmitters.

\section{Improved System Implementation and Extended Experimental Results}

Based on the above considerations, an improved and extended version of the system has been implemented. In this version, the frequency separation operating mode is selected, due to its favorable measurement update rate property. The system architecture, which is shown in Fig. 13, is comprised of four known-position transmitters operating at the frequencies given in Section VI.B. The receiver uses an Agilent U2331 A data acquisition (DAQ) board, connected to a PC for measurement automation and data processing. A picture of the system in the experimental laboratory environment is shown in Fig 14.

Using this setup, the receiver can acquire the signal and estimate the amplitude of each sinusoidal component simultaneously, using processing methods based on the Discrete Fourier Transform (DFT). In particular, as shown in Fig. 15, a data record $x[n]$ obtained with sampling rate 3 $\mathrm{MSa} / \mathrm{s}$ and having length $2^{20} \mathrm{Sa}$ is acquired by the DAQ and processed using the FFT algorithm. A flattop window is used because it allows for accurate amplitude estimation by overcoming scalloping loss [18]0.

Experimental results obtained with the improved system setup are shown in Fig. 16 and 17. After performing the 
calibration procedure described in Section IV, the mobile receiver node was placed in 46 points along the trajectory in Fig. 16, which includes both favorable and unfavorable geometric configurations. The test area is larger than that considered above. Specifically, the transmitters are placed in the four corners of a $4.5 \times 4.5 \mathrm{~m}^{2}$ square, the maximum transmitter-receiver distance is approximately $6.3 \mathrm{~m}$, and the receiver trajectory spans an area which is $7 \mathrm{~m}$ long and $3 \mathrm{~m}$ wide. At each point, a data record was acquired and the position was measured based on DFT amplitude estimation. The resulting average positioning error is $10.5 \mathrm{~cm}$, and the empirical Cumulative Distribution Function (CDF) of the error is shown in Fig. 17. Results show that the error is less than 30 $\mathrm{cm}$ in $90 \%$ of the cases.

Finally, additional experiments were conducted to assess the sensitivity with respect to deviations from coplanarity of the coils. Results show that, in the considered experimental setup, when the receiver is placed at a height of approximately $50 \mathrm{~cm}$ above the plane defined by the transmitting coils, the 2D positioning error increases by about $5 \mathrm{~cm}$ with respect to the coplanar position. Therefore, the system is proven to be relatively insensitive to deviations from coplanarity, and thus is practical for 2D indoor positioning applications.

\section{CONCLUSION}

In this paper, we described the design approach for a novel position-measurement system based on AC artificially generated magnetic fields in indoor environments. The system has the potential of overcoming some of the main drawbacks of the current state-of-the-art solutions, including multipath effects, line-of-sight requirements and limited operational range.

We presented the results of numerical simulations for the computation of the mutual inductance, and evaluated the feasibility of the proposed approach by experimental tests on a prototype. The realized system exhibits a maximum positioning error of less than $10 \mathrm{~cm}$ in an indoor environment over a $3 \times 3 \mathrm{~m}^{2}$ area, and an average error below $20 \mathrm{~cm}$ in less favorable conditions. The realized system, used in frequency separation operating mode, and combined with digital signal processing, provides flexibility and accuracy for achieving robust indoor positioning.

\section{APPENDIX A}

In [13], the set of equations needed to calculate (4) is provided, assuming the following definitions:

$$
\begin{gathered}
\alpha=\frac{R_{S}}{R_{P}}, \quad \beta=\frac{x_{C}}{R_{P}}, \quad \gamma=\frac{y_{C}}{R_{P}}, \quad \delta=\frac{z_{C}}{R_{P}} \\
\ell=\sqrt{a^{2}+b^{2}}, \quad L=\sqrt{a^{2}+b^{2}+c^{2}} \\
p_{1}= \pm \frac{\gamma_{C}}{\ell}, \quad p_{2}=\mp \frac{\beta \ell^{2}+\gamma a b}{\ell L}, \quad p_{3}=\frac{\alpha c}{L}, \\
p_{4}=\mp \frac{\beta a b-\gamma \ell^{2}+\delta b c}{\ell L}, \quad p_{5}=\mp \frac{\beta c-\delta a}{\ell} \\
A_{0}=1+\alpha^{2}+\beta^{2}+\gamma^{2}+\delta^{2}+2 \alpha\left(p_{4} \cos \varphi+p_{5} \sin \varphi\right)
\end{gathered}
$$

$$
\begin{aligned}
& V_{0}^{2}=\alpha^{2}\left[\left(1-\frac{b^{2} c^{2}}{\ell^{2} L^{2}}\right) \cos ^{2} \varphi+\frac{c^{2}}{\ell^{2}} \sin ^{2} \varphi+\frac{a b c}{\ell^{2} L} \sin 2 \varphi\right] \\
& +\beta^{2}+\gamma^{2} \mp 2 \alpha \frac{\beta a b}{\ell L} \cos \varphi \mp \frac{2 \alpha \beta c}{\ell} \sin \varphi \\
& k=\sqrt{\frac{4 V_{0}}{A_{0}}} \quad \Psi(k)=\left(1-\frac{k^{2}}{2}\right) K(k)-E(k)
\end{aligned}
$$

where $\left(x_{C}, y_{C}, z_{C}\right)$ denotes the center of the secondary coil, and $K(k)$ and $E(k)$ denote the complete elliptic integrals of the first and second kind respectively [13].

\section{REFERENCES}

[1] Pahlavan, K., Li, X., and Makela, J. P., "Indoor geolocation science and technology," IEEE Communications Magazine, 40(2), pp. 112-118, 2002.

[2] Patwari, N., Ash, J. N., Kyperountas, S., Hero III, A. O., Moses, R. L., and Correal, N. S., "Locating the nodes: cooperative localization in wireless sensor networks.” IEEE Signal Processing Magazine, 22(4), pp. 54-69, 2005.

[3] Cazzorla, A., De Angelis, G., Moschitta, A., Dionigi, M., Alimenti, F., and Carbone, P., "A 5.6-GHz UWB Position Measurement System," IEEE Transactions on Instrumentation and Measurement, vol.62, no.3, pp.675-683, March 2013.

[4] Santinelli, G., Giglietti, R., and Moschitta, A., "Self-calibrating indoor positioning system based on ZigBee ${ }^{\circledR}$ devices," IEEE Instrumentation and Measurement Technology Conference (I2MTC), pp.1205-1210, 5-7 May 2009.

[5] Mautz, R., "Indoor Positioning Technologies, Swiss Geodetic Commission," Geodetic-Geophysical Reports of Switzerland, no. 86, 2012.

[6] Polhemus Fastrak Motion Tracking System. [Online]. Available: http://www.polhemus.com/?page=motion_fastrak, retrieved 2014 .

[7] Blankenbach, J., and Norrdine, A., "Position estimation using artificial generated magnetic fields," in IEEE International Conference on Indoor Positioning and Indoor Navigation (IPIN), September 2010.

[8] Blankenbach, J., Norrdine, A., and Hellmers, H., "A robust and precise $3 \mathrm{D}$ indoor positioning system for harsh environments," in IEEE International Conference on Indoor Positioning and Indoor Navigation (IPIN), November 2012.

[9] Dionigi, M., De Angelis, G., Moschitta, A., Mongiardo, M., and Carbone, P., "A simple ranging system based on mutually coupled resonating circuits". IEEE Transactions on Instrumentation and Measurement, vol.63, no.5, pp.1215-1223, May 2014.

[10] De Angelis, A., Dwivedi, S., Handel, P., Moschitta, A., and Carbone, P., "Ranging results using a UWB platform in an indoor environment," IEEE International Conference on Localization and GNSS (ICL-GNSS), 25-27 June 2013.

[11] R. Koma, S. Nakamura, S. Ajisaka, and H. Hashimoto, "Basic Analysis of the Circuit Model Using Relay Antenna in Magnetic Resonance Coupling Position Sensing System," IEEE/ASMA International Conference on Advanced Intelligent Mechatronics (AIM2011), Budapest, Hungary, July 3-7, 2011.

[12] G. De Angelis, A. De Angelis, M. Dionigi, M. Mongiardo, A. Moschitta, P. Carbone, "An Accurate Indoor Position-Measurement System Using Mutually Coupled Resonating Circuits" In IEEE International Instrumentation and Measurement Technology Conference (I2MTC), Montevideo, Uruguay, May 2014.

[13] Babic, S., Sirois, F., Akyel, C., and Girardi, C., "Mutual inductance calculation between circular filaments arbitrarily positioned in space: alternative to Grover's formula," IEEE Transactions on Magnetics, 46(9), pp. 3591-3600, September 2010.

[14] Trotter, M.S., Ricketts, D.S., Griffin, J.D., "Experimental demonstration of complex image theory for vertical magnetic dipoles with applications to remote sensing and position tracking, "IEEE International Conference 
on Electromagnetics in Advanced Applications (ICEAA), pp.1248,1251, 9-13 Sept. 2013.

[15] Prigge, E. A. and How, J. P. "Signal architecture for a distributed magnetic local positioning system," IEEE Sensors Journal, 4(6), 864873, 2004.

[16] J. Rantakokko, J. Rydell, P. Strömback, P. Händel, J. Callmer, D. Törnqvist, F. Gustafsson, M. Jobs, M. Grudén, "Accurate and reliable soldier and first responder indoor positioning: multisensor systems and cooperative localization," IEEE Wireless Communications, vol.18, no.2, pp.10-18, April 2011.

[17] Lee T.H., The Design Of CMOS Radio-Frequency Integrated Circuits, Cambridge University Press, 1998, Chapter 11.

[18] P. Carbone, E. Nunzi, D. Petri, "Frequency-domain-based least-squares estimation of multifrequency signal parameters," IEEE Transactions on Instrumentation and Measurement, vol.49, no.3, pp.555-558, Jun 2000.

L. Salvatore, A. Trotta, "Flat-top windows for PWM waveform processing via DFT," IEE Proceedings Electric Power Applications, part B, vol.135, no.6, pp.346-361, Nov 1988. 